\title{
Analysis of Copolymers by Laser Desorption Fourier Transform Mass Spectrometry
}

\author{
Lydia M. Nuwaysir and Charles L. Wilkins \\ Department of Chemistry, University of California at Riverside, Riverside, California, USA \\ William J. Simonsick, Jr. \\ E.I. duPont de Nemours \& Co., Inc., Marshall R\&D Laboratory, Philadelphia, Pennsylvania, USA
}

\begin{abstract}
A series of methylmethacrylate/butylacrylate, methylmethacrylate/styrene, and poly(ethylene glycol)/poly(propylene glycol) copolymers were analyzed by laser desorption Fourier transform mass spectrometry. Molecular weight distributions and overall copolymer compositions were determined. Typical spectra arise from a distribution of alkali metal cationized oligomer ions reflecting copolymer composition. Number- and weight-average molecular weights were calculated for each category of copolymer series as well as for overall copolymer mixtures. In addition, mass spectra were analyzed to estimate copolymer monomer contributions; these results were compared with manufacturer feed mix ratios as a test of the relative accuracy of the analysis. Although a dual-cell Fourier transform mass spectrometer was used, all copolymer analyses reported were carried out as single (source) cell measurements to ensure that no mass discrimination would occur. A series of Fourier transform mass spectrometry dual-cell experiments also were performed to evaluate the effect of ion transfer time on molecular weight averages and compositions. As expected, mass discrimination occurred when short transfer times and analyzer cell detection were employed. Under these conditions, molecular weight averages varied by more than $50 \%$ from values obtained in the single-cell measurements. (] Am Soc Mass Spectrom 1990, 1, 66-71)
\end{abstract}

$\mathrm{T}$ The widespread utility of polymeric materials in so many applications stems from the ability to manipulate and control certain physical and chemical properties to meet specific performance requirements. Different applications require polymers with different degrees and combinations of adhesion, viscosity, hardness, resiliency, stability, flexibility, rigidity, and crack resistance; these requirements are often in conflict with one another. Copolymerization of two or more monomers provides a convenient method of synthesizing materials with the desired properties. Through manipulation of the copolymer composition, monomer structure, and molecular dimensions, the desired characteristics often can be produced.

A number of analytical methods have been developed to characterize polymer mixtures. Chromatographic methods such as size exclusion chromatography (gel permeation chromatography) are among the most prevalent techniques for analyzing polymer composition and molecular weight distribution. Size exclusion chromatography has been particularly beneficial for monitoring polymer syntheses by providing rapid analysis of samples at various stages of conversion [1]. Under ideal conditions, polymer molecules frac-

Address reprint requests to Charles L. Wilkins, Department of Chemistry, University of California at Riverside, Riverside, CA 92521, USA. tionate according to their size in solution. Molecular weight information obtained from the chromatogram is highly dependent upon the accuracy of the calibration procedure. Well-characterized, narrow molecular weight distribution polymer calibrants of similar chemical composition must be used to provide the most accurate results. Even with these precautions, fractionation of copolymers frequently leads to anomalous results owing to the differences in the chemical nature of the monomers [2]. Significant differences in polymer-solvent packing interactions result in separations that are not strictly by size or composition and lead to errors in estimation of molecular weight. Additional errors can arise from nonlinear detector response with respect to various copolymer compositions.

Pyrolysis mass spectrometry methods can be used to analyze all types of polymers, often providing highly reproducible "fingerprint" spectra [2-4]. These spectra are useful for structural identification, isomer differentiation, and copolymer composition but are inadequate for molecular weight characterization. Alternatively, mass spectra obtained using soft ionization techniques such as laser desorption (LD), fast atom bombardment (FAB), secondary ion mass spectrometry (SIMS), and potassium ionization of desorbed species $\left(\mathrm{K}^{+} \mathrm{IDS}\right)$ frequently reveal minimal fragmentation and are more indicative of polymer molecular weight dis- 
tributions [5-18]. These recent improvements in mass spectrometric techniques for volatilization and ionization of intact macromolecules led to renewed interest in the use of mass spectrometry for detailed polymer analysis. A polymer mass spectrum of intact oligomer ions with a minimal number of fragment ions preserves molecular weight information and allows determination of absolute molecular weight distributions (MWD). Number-average $\left(\bar{M}_{n}\right)$ and weightaverage $\left(\bar{M}_{\mathrm{w}}\right)$ molecular weights can be calculated from these spectra using eqs. 1 and 2 , in which $N_{i}$ is the number of moles of molecules of molecular weight $M_{i}$ in a particular polymer mixture.

$$
\begin{gathered}
\bar{M}_{\mathrm{n}}=\sum N_{i} M_{i} / \sum N_{i} \\
\bar{M}_{\mathrm{w}}=\sum N_{i} M_{i}^{2} / \sum N_{i} M_{i}
\end{gathered}
$$

Application of soft ionization methods to mass spectrometry of copolymers not only allows determination of overall molecular weight averages, but also permits direct examination of individual copolymer compositions and distributions. $\bar{M}_{\mathrm{n}}$ and $\bar{M}_{\mathrm{W}}$ values for each particular type of copolymer can be calculated, as well as the contributions of each type to the total copolymer mixture. Individual monomer contributions were estimated by summing the weighted contributions to each of the mass spectrometrically detected ions over all the oligomer ion series for a particular starting monomer ratio [e.g., over all five ion series for MMA/BA-90/10 (see under Experimental)]. If results are to be accurate, volatilization, sample transmission to analyzer, and mass analysis must all be free of mass discrimination. For single-cell measurements, laser desorption Fourier transform mass spectrometry (LD/FTMS) meets these requirements. Previous studies have shown that molecular weight distributions and molecular weight averages calculated from LD/FTMS data for various polar polymer samples are in good agreement with those obtained from independent alternative analytical methods [13, 14]. For example, $\bar{M}_{\mathrm{n}}$ values calculated for poly(ethylenimine) polymers analyzed by LD/FTMS were consistent with nominal molecular weights provided by the manufacturer, while field desorption, FAB, and electrohydrodynamic (EH) ionization mass spectrometric results were substantially lower [13]. A follow-up study investigated some of the factors influencing $\mathrm{EH}$ mass spectra of poly(ethylenimines) [19]. In the present study, analysis of a variety of copolymers was carried out with a dual-cell Fourier transform mass spectrometer in order to evaluate the capability of such an instrument operated in a single-cell analysis mode to accurately analyze copolymer mixtures.

When dual-cell FTMS experiments are performed, ionization and detection events are spatially separated by using two cells separated by a common solid trap- ping plate with a small (typically 2-mm diameter) conductance-limiting orifice for transmission of ions. Ions are formed in the source cell and are allowed to transfer to the analyzer cell by grounding the center trap plate [20]. The trapping motion along the $z$ axis (parallel to the magnetic field) is a function of the trap plate voltages, magnetic field strength, and mass-tocharge ratio [21]. In particular, the frequency of oscillation along the $z$ axis is inversely proportional to $\mathrm{m} / \mathrm{q}$, and hence transfer time from one cell to another is proportional to ion mass. This dependency can result in mass discrimination under certain conditions $[22,23]$ and can be evaluated by examining the changes in ion abundances as a function of transfer time for analyzer cell detection. Because the single-cell measurements provide reliable determinations of copolymer distributions, it is of interest to compare the outcome of transfer time-dependent analyzer cell detection to observe the effects on the copolymer ion distribution from mass discrimination arising from the ion transfer process. These effects can be determined quantitatively by computing molecular weight averages and copolymer compositions for the transferred copolymer ion populations and comparing these results with those reflecting the true copolymer distributions (as determined from source cell detection measurements).

\section{Experimental}

Mass spectra were recorded using a Nicolet 2000 Fourier transform mass spectrometer interfaced with a Tachisto 215 pulsed $\mathrm{CO}_{2}$ laser. The spectrometer is equipped with a 7-Tesla superconducting magnet and solids autoprobe and contains differentially pumped source and analyzer cells separated by a 2-mm conductance limit. The $\mathrm{CO}_{2}$ laser is directed into the mass spectrometer through a quartz window in the source flange and is focused onto the tip of the solids probe to $\sim 1 \mathrm{~mm}^{2}$ spot size by an off-axis paraboloid mirror mounted on the source cell assembly. The laser delivers approximately $10^{6}-10^{8} \mathrm{~W} / \mathrm{cm}^{2}$ per $80 \pm 40$-ns pulse.

Spectra were obtained in the direct mode under broadband excitation and observation conditions. Source cell detection was used for copolymer analysis to avoid mass discrimination due to ion transfer. In addition, a series of analyzer cell experiments were performed with various transfer times to evaluate the effects of mass discrimination on the apparent copolymer composition. Transfer times corresponded to the length of time the conductance limit was grounded to allow passage of laser-desorbed ions from the source cell into the analyzer cell. Trapping voltages of $1.6 \mathrm{~V}$ were used for both source cell and analyzer cell experiments. Following a delay of 5-10 s after the desorption event to allow desorbed neutral species to be pumped away, ions were excited via a $200-\mathrm{Vpp}$ chirp excite from 0 to $200 \mathrm{kHz}$ at $180 \mathrm{~Hz} / \mu \mathrm{s}$, from 0 to 800 $\mathrm{kHz}$ at $400 \mathrm{~Hz} / \mu \mathrm{s}$, or from 0 to $1000 \mathrm{kHz}$ at $900 \mathrm{~Hz} / \mu \mathrm{s}$. $64 \mathrm{~K}$ data transients were collected, augmented by one 
level of zero filling, and fast Fourier transformed to obtain magnitude mode spectra. No apodization or signal averaging was employed. For calculating weight averages and distributions, the relative ion abundances were estimated from peak heights by using the intensity value associated with the maximum data point in the peak. Calculations for each oligomer ion included all isotopic contributions. Although numerous replicate analyses were performed and all were in excellent qualitative agreement, no statistical analysis of reproducibility was done.

Methylmethacrylate/butylacrylate copolymers and methylmethacrylate/styrene copolymers were obtained from Marshall $R$ \& $D$ Laboratory (E. I. duPont de Nemours \& Co., Inc., Philadelphia, PA). Specific copolymers studied are composed of monomer weight percentage ratios of 90:10, 80:20, and 70:30 methylmethacrylate to butylacrylate or styrene and are designated as MMA/BA-90/10, MMA/BA-80/20, MMA/BA-70/30, MMA/STY-90/10, MMA/STY-80/20, and MMA/STY-70/30. Gel permeation chromatography (GPC) was carried out using a four-column set of $10^{5} \mathrm{~A}, 10^{4} \mathrm{~A}, 10^{3} \mathrm{~A}$, and $10^{2} \mathrm{~A}$ Ultrastyragel GPC columns (Waters, Milford, MA) and tetrahydrofuran as the mobile phase. Amine-terminated poly(ethylene glycol)/poly(propylene glycol) copolymers of approximate molecular weights 600,900 , and 2000 (Jeffamine ED-600, ED-900, and ED-2001) were obtained from Texaco Chemical Company, Houston, Texas. MMA/BA and MMA/STY copolymers are low vapor pressure viscous liquids; Jeffamine ED-600 and ED-900 are lowviscosity, low vapor pressure liquids; and Jeffamine ED-2001 is a waxy solid. Samples were introduced either neat, by depositing a few microliters onto a stainless steel probe tip, or by dissolving them in methanol, spotting a few drops of the resulting solutions onto the probe tip, and evaporating the solvent. For the MMA/STY-70/30 sample, $\mathrm{KCl}$ was added to the methanol solutions to increase the yield of potassium ion-attached oligomers. After insertion of the sample probe into the mass spectrometer source, background pressure was allowed to drop to about $2-3 \times 10^{-8}$ torr before analysis.

\section{Results and Discussion}

Figure 1a contains the positive-ion LD/FTMS spectrum for MMA/BA-80/20. This copolymer spectrum consists of a very complex array of peaks. The polymer ion envelope extends from $\mathrm{m} / \mathrm{z} 600$ to above $\mathrm{m} / \mathrm{z} 3500$. Closer inspection of the expanded mass region between $\mathrm{m} / \mathrm{z} 1390$ and $\mathrm{m} / \mathrm{z} 1890$ reveals several series of ions (Figure 1b). All ions are potassium-attached and, for convenience, are designated by the general formula $\left[(\mathrm{MMA})_{m}(\mathrm{BA})_{n}+\mathrm{K}\right]^{+}$. The terminal end group is unsaturated, and so the oligomer ions appear at two mass units lower than the general formula implies. Ions are assigned and identified by their $m / n$ labels, which represent the number of methylmethacrylate $(m)$ and
A

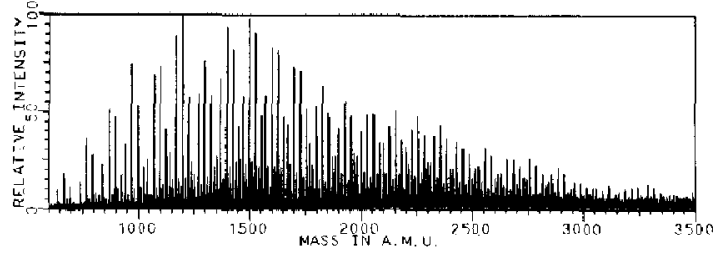

B

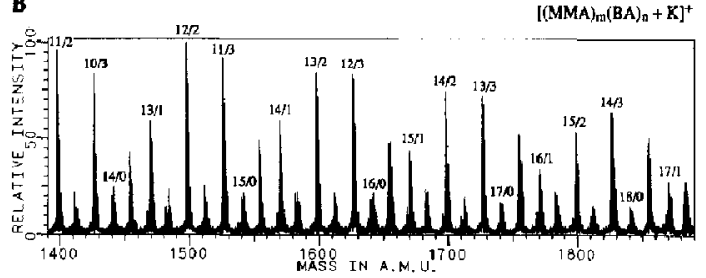

Figure 1. Laser desorption Fourier transform mass spectrum of MMA/BA-80/20. (a) Full mass scale; (b) expanded mass scale. Compositions of oligomer ions are identified by twin labels.

butylacrylate (n) units for each particular oligomer ion. All copolymer oligomer ions contain various numbers of methylmethacrylate repeating units. A homopolymer distribution for ions of the form [(MMA) $m+K]^{+}$ (labeled $m / 0$ in Figure 1b) also is observed. Superimposed over the homopolymer pattern are the patterns arising from copolymer oligomer ion series containing from one to five butylacrylate units associated with the methylmethacrylates. For clarity, Figure $1 \mathrm{~b}$ is labeled only up to the $m / 3$ series. These series are observed for all three MMA/BA copolymers. Table 1 summarizes molecular weight averages, compositions, and distributions determined from the LD/FT mass spectra of the copolymers examined. Note that relative abundances of the ions within each series varics widely among the different copolymers. This is illustrated in Figure 2, which shows the expanded mass region plots between $m / 2920$ and 1720 for the LD mass spectra of MMA/BA-90/10, $-80 / 20$, and $-70 / 30$ (Figure 2a-c). Labels indicate the number of butylacrylate moieties associated with nine methylmethacrylates $\left[(\mathrm{MMA})_{9}(\mathrm{BA})_{n}+\mathrm{K}\right]^{+}$. It is obvious that the abundances of ions with greater numbers of butylacrylates increase as the weight percentage of butylacrylate in the copolymer is increased. For MMA/BA-90/10, the oligomer ion series contributing the most to the total ion current (31\%) arises from the $\mathrm{MMA}_{m} \mathrm{BA}_{1}$ series from among five detected above a $10 \%$ minimum ion abundance threshold $(m / 0, m / 1, m / 2, m / 3$, and $m / 4)$. In Figure $2 a$, the $\left[(\mathrm{MMA})_{9}(\mathrm{BA})_{n}+\mathrm{K}^{+}\right.$distribution contains a maximum abundance of ions for $n=1$. The MMA/BA80/20 distribution is shifted to higher masses (Figure $2 \mathrm{~b}$ ), and the maximum abundance of ions is observed for the $n=2$ series. For this sample, seven oligomer series with $n=0$ to $n=6$ are observed. Similarly, MMA/BA-70/30 contains seven series of oligomer ions with $n=0$ to $n=6$ and maximum ion abundance for the $n=3$ series. Molecular weight averages increase 


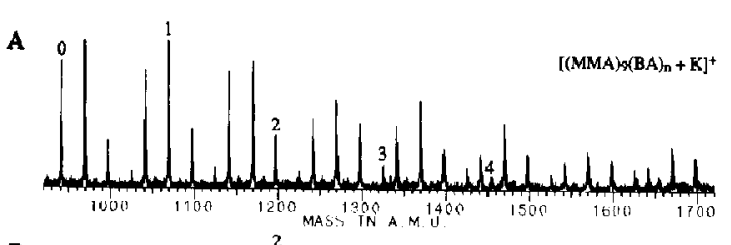

$\mathbf{8}$

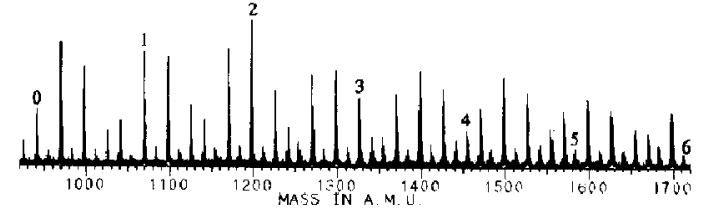

C

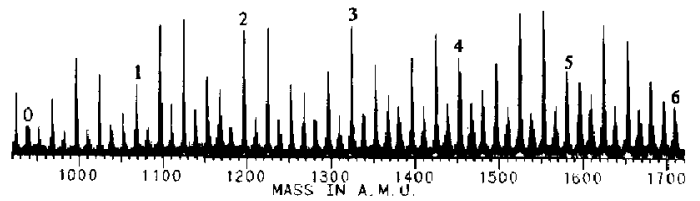

Figure 2. Expanded mass regions of the LD/FTMS spectra of MMA/BA copolymers, lon labels correspond to the number, $n_{r}$ of BAs associated with nine MMAs: (a) MMA/BA-90/10; (b) MMA/BA-80/20; (c) MMA/BA-70/30.

from MMA/BA-90/10 to MMA/BA-70/30, and the polydispersity of all three copolymer mixtures is near 1.0, indicating fairly uniform polymer distributions.

Both $\mathrm{K}^{+}$- and $\mathrm{Na}^{+}$-attached oligomer ions are observed in the LD/FTMS spectra of MMA/STY copolymers. The spectrum in Figure 3 displays the MMA/STY copolymer distribution for MMA/STY90/10. The most abundant ions arise from the three series $\left[(\mathrm{MMA})_{m}+\mathrm{Cat}^{+},\left[(\mathrm{MMA})_{m}(\mathrm{STY})_{1}+\right.\right.$ Cat $^{+}{ }^{+}$, and $\left[(\mathrm{MMA})_{m}(\mathrm{STY})_{2}+\mathrm{Cat}^{+}{ }^{+}\right.$, where Cat represents either $\mathrm{Na}^{+}$or $\mathrm{K}^{+}$attachment. These series extend from $m / z 630$ to $m / z 3900$, with the most abundant ions observed for the $\left[(\mathrm{MMA})_{m}(\mathrm{STY})_{1}+\mathrm{K}^{+}{ }^{+}\right.$serics. $\Lambda$ ddition of potassium salts to the sample provides spectra dominated by $\mathrm{K}^{+}$-attached oligomer ions and facilitates the copolymer analysis. Similar ion series are observed in the spectra of MMA/STY-80/20 and MMA/STY-70/30. The abundances of ions within particular copolymer compositions are different for each sample, however, and reflect the shift in sample monomer ratios.

Because of the widespread use of GPC for polymer analyses, it is of interest to compare the present LD/FTMS analyses with GPC results. For all of the methylmethacrylate copolymers analyzed, the manufacturer provided GPC weight- and number-average molecular weights and polydispersities for the samples. As mentioned previously, the accuracy of such chromatographic procedures is critically dependent upon the use of appropriate well-characterized standards as calibrants. For the data summarized in Table 2 , narrow molecular weight range polystyrene standards were used because of unavailability of chemically similar copolymer standards. As is obvious, the GPC data averages and polydispersities are different from the directly measured values obtained by LD/FTMS. Because LD/FTMS provides a direct measurement of the oligomer ion distribution, it could offer a possible solution to the common dilemma of a lack of wellcharacterized copolymer standards when chromatographic analyses are employed. Perhaps LD/FTMS could be used to characterize standards appropriate for accurate GPC analysis of copolymers. There is precedent for this suggestion, because the $\mathrm{K}^{+}$IDS technique [16-18] was used previously to calibrate GPC for routine analysis of low molecular weight polymers (MW < 1000). Comparisons of $\mathrm{K}^{+}$IDS and GPC quantitative data showed that they agreed within $10 \%$ or better [16]. Molecular weight and compositional information available by LD/FTMS also allows evaluation of the accuracy of computer polymerization models commonly employed to simulate polymer growth.

Jeffamine samples are poly(oxyethylene)diamines derived from propylene oxide-capped poly(ethylene glycol). The general formula for these compounds is $\mathrm{H}_{2} \mathrm{NCH}\left(\mathrm{CH}_{3}\right) \mathrm{CH}_{2}-\left(\mathrm{OCH}\left(\mathrm{CH}_{3}\right) \mathrm{CH}_{2}\right)_{a}-\left(\mathrm{OCH}_{2} \mathrm{CH}_{2}\right)_{b}-$ $\left(\mathrm{OCH}_{2} \mathrm{CH}\left(\mathrm{CH}_{3}\right)\right)_{c}-\mathrm{NH}_{2}$. For simplicity, the oligomer ions are designated by the empirical formula $\mathrm{R}(\mathrm{PPG})_{m}(\mathrm{PEG})_{n}$ where $\mathrm{R}=\mathrm{C}_{3} \mathrm{H}_{10} \mathrm{~N}_{2}$. Figure 4 contains the LD/FTMS spectrum for Jeffamine ED-900 of

Table 1. Copolymer molecular weight averages, distributions, and compositions

\begin{tabular}{|c|c|c|c|c|c|}
\hline Copolymer & $\bar{M}_{\mathrm{n}}$ & $\bar{M}_{\mathrm{w}}$ & $D^{\mathrm{a}}$ & $\begin{array}{l}\text { No. of } \\
\text { series }^{b}\end{array}$ & Calc. monomer ratio (\%) \\
\hline MMA/BA-90/10 & 1274 & 1449 & 1.14 & 5 & $88.3 / 11.7$ \\
\hline MMA/BA-80/20 & 1292 & 1395 & 1.08 & 7 & $83.7 / 16.3$ \\
\hline MMA/BA-70/30 & 1694 & 1882 & 1.11 & 7 & $81.6 / 18.4$ \\
\hline MMA/STY-90/10 & 1703 & 1892 & 1.11 & 3 & $93.7 / 6.3$ \\
\hline MMA/STY $80 / 20$ & 1145 & 1243 & 1.09 & 4 & $83.6 / 16.4$ \\
\hline MMA/STY-70/30 & 1289 & 1559 & 1.21 & 6 & $78.5 / 21.5$ \\
\hline Jeffamine-ED 600 & 633 & 655 & 1.04 & 4 & $-c$ \\
\hline Jeffamine-ED900 & 956 & 971 & 1.02 & 4 & - \\
\hline Jeffamine-ED2001 & 1910 & 1934 & 1.01 & 4 & - \\
\hline
\end{tabular}

a Polydispersity $\left(\bar{M}_{w} / \bar{M}_{n}\right)$.

- Number ol dilferent oligumer series with a rrinimum ion abundance threshold of $10 \%$.

a Not determined for Jeffamine samples. 


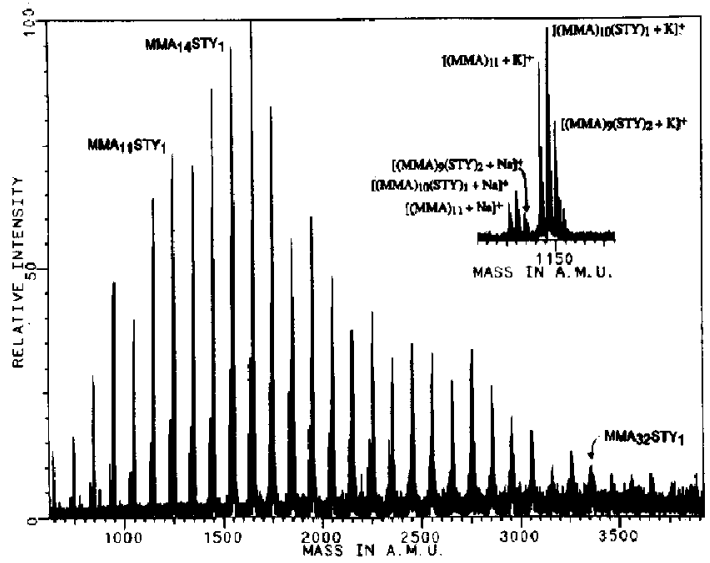

Figure 3. Laser desorption Fourier transform mass spectrum of MMA/STY-90/10. Inset: Compositions of ions are identified for a representative mass region.

average molecular weight 900 . Four different copolymer compositions are apparent from the four series $\left[\mathrm{R}(\mathrm{PPG})_{1}(\mathrm{PEG})_{n}+\mathrm{H}\right]^{+},\left[\mathrm{R}(\mathrm{PPG})_{2}(\mathrm{PEG})_{n}+\mathrm{H}\right]^{+}$, $\left[\mathrm{R}(\mathrm{PPG})_{3}(\mathrm{PEG})_{n}+\mathrm{H}\right]^{+}$, and $\left[\mathrm{R}(\mathrm{PPG})_{4}(\mathrm{PEG})_{n}+\mathrm{H}\right]^{+}$, in which one, two, three, and four PPGs, respectively, are associated with a variable number of PEGs. Jeffamines ED-600 and ED-2001 also contain protonated and $\mathrm{K}^{+}$-attached ions representative of these same series. Weight averages calculated from the LD/FTMS spectra agree with approximate values provided by the manufacturer.

Figure 5 contains a series of MMA/BA-90/10 spectra obtained after transferring ions to the analyzer cell immediately following formation for subsequent excitation and detection. The six spectra differ in the amount of time allowed for the transfer of ions between the source and analyzer cells (i.e., the length of

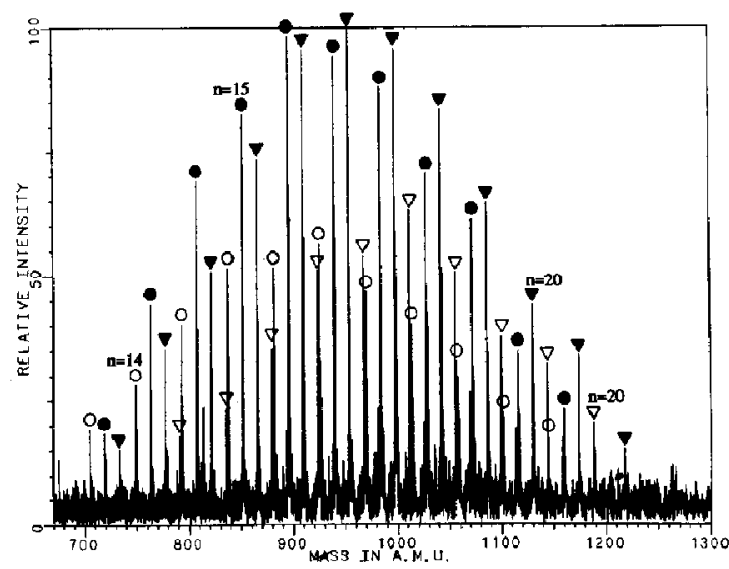

Figure 4. Laser desorption Fourier transtorm mass spectrum of Jeffamine ED-900. Labels designate copolymer compositions for ions with the empirical formula $\left[\mathrm{R}(\mathrm{PPG})_{m}(\mathrm{PEG})_{n}+\mathrm{H}\right]^{+}$, where $n=$ the number of PEGs associated with $\bigcirc-1, \bullet-2, \nabla=$ 3 , or $\nabla=4$ PPGs.
Table 2. Molecular weight averages from GPC data

\begin{tabular}{lrrl}
\hline Copolymer & $\bar{M}_{\mathrm{n}}$ & $\bar{M}_{\mathrm{w}}$ & $D^{\mathrm{a}}$ \\
\hline MMA/BA-90/10 & 590 & 990 & 1.7 \\
MMA/BA-80/20 & 750 & 1300 & 1.7 \\
MMA/BA-70/30 & 900 & 1800 & 2.0 \\
MMA/STY-90/10 & 1100 & 2300 & 2.1 \\
MMA/STY-80/20 & 980 & 2100 & 2.1 \\
MMA/STY-70/30 & 1800 & 4400 & 2.4 \\
\hline
\end{tabular}

a Polydispersity $\left(\vec{M}_{w} / \vec{M}_{n}\right)$

time the conductance limit was grounded). Ion partitioning is graphically illustrated by the modulation of the copolymer oligomer ion spectra apparent in Figure $5 a-f$. Table 3 contains apparent molecular weight averages and copolymer distributions calculated from these spectra. For analyzer cell analysis, these data clearly
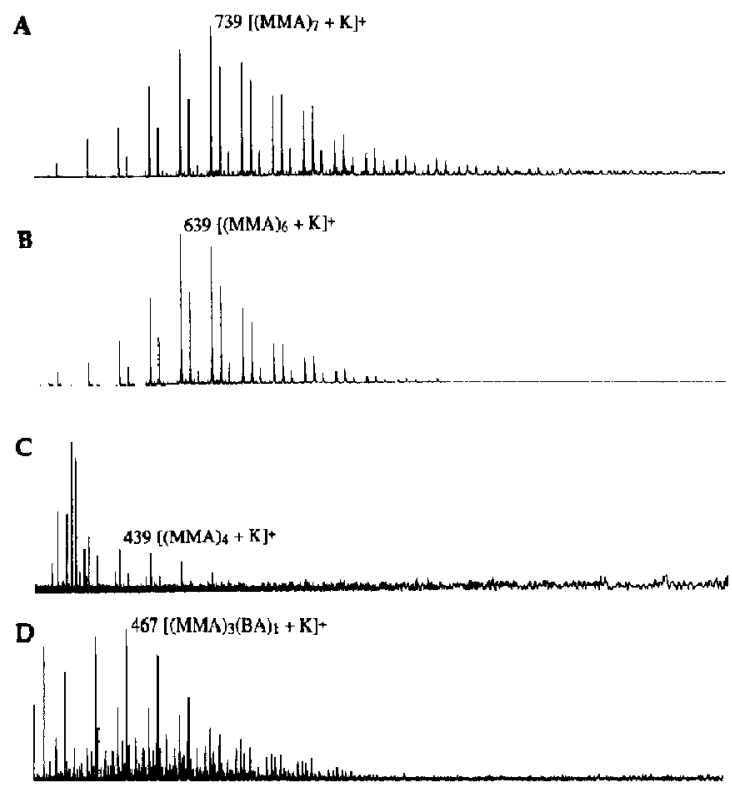

$\mathbf{E}$

$867[(\mathrm{MMA}) \gamma(\mathrm{BA}) \mid+\mathrm{K}]^{+}$

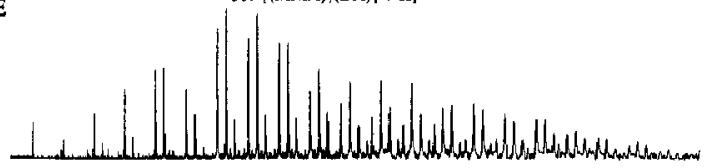

F

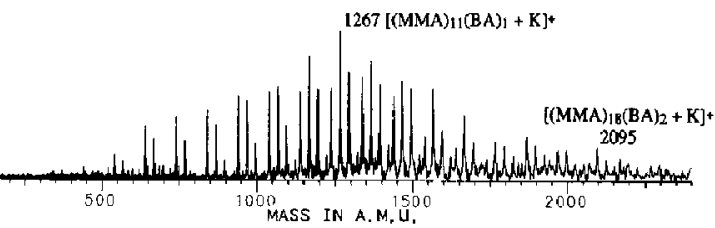

Figure 5. Laser desorption mass spectra of MMA/BA-90/10. Ions were transferred to the analyzer cell before excitation and detection. Transfer times correspond to the length of time the conductance limit was grounded: (a) $400 \mu \mathrm{s}$; (b) $600 \mu \mathrm{s}$; (c) $800 \mu \mathrm{s}$; (d) 1 ms; (e) $1.2 \mathrm{~ms}$; (f) $1.4 \mathrm{~ms}$. Mass scale is identical for all spectra. 
Table 3. MMA/BA-90/10 apparent weight averages and distributions as a function of ion transfer time

\begin{tabular}{lrrrrrrr}
\hline & & & & \multicolumn{3}{c}{$\% \mathrm{MMA}_{m} \mathrm{BA}_{n}{ }^{3}$} \\
\cline { 6 - 8 } Transfer time $(\mu \mathrm{s})$ & $\bar{M}_{n}$ & $\bar{M}_{w}$ & $D$ & $n=0$ & $n-1$ & $n-2$ \\
\hline 400 & 868 & 972 & 1.12 & 48 & 40 & 12 \\
600 & 752 & 793 & 1.05 & 52 & 41 & 7 \\
800 & 601 & 921 & 1.53 & 66 & 28 & 6 \\
1000 & 691 & 770 & 1.11 & 55 & 35 & 10 \\
1200 & 1045 & 1273 & 1.22 & 41 & 41 & 18 \\
1400 & 1141 & 1355 & 1.19 & 39 & 41 & 20 \\
\hline
\end{tabular}

a For cach value of $n$, the abundances of the oligomeric series of ions for all values of $m$ are added. Table entries represent relative contributions to the tatal ion current.

demonstrate that choice of ion transfer time is critical if accurate results are to be obtained. Comparison of these data with the source cell analysis of the same sample (Table 1), which is regarded as reflecting the actual composition of the copolymer mixture, reveals that short transfer times result in serious mass discrimination. This is easily understood as arising from the mass-dependent transfer rate for dual-cell ion partition, as previously documented $[22,23]$. Lower mass ions, with correspondingly higher transverse oscillation frequencies, achieve equipartition more rapidly than more massive ions. However, as these data show, it is possible to achieve accurate results by the simple expedient of choosing a sufficiently long transfer time to ensure equilibrium ion partition. Thus, when a partition time of $1400 \mu \mathrm{s}$ is chosen, the measured results approach those obtained for source cell analysis.

\section{Acknowledgments}

Partial support for this research by the Shell Development Company and by the National Science Foundation under grant NSF CHE-89-11685 is gratefully acknowledged.

\section{References}

1. Pavelich, W. A.; Livigni, R. A. J. Polym. Sci., Part C 1968, 21. 215-223.

2. Garcia Rubio, L. H.; McGregor, J. F.; Hamielec, A. E. In Polymer Characterization; Craver, C. D., Ed.; American Chemical Society, Washington, DC, 1983; pp 311-344.

3. Garozzo, D.; Giuffrida, M.; Montaudo, G,; Lenz, R, W. J. Polym. Sci., Part A 1987, 25, 271-284.
4. Montaudo, G.; Scamporrino, E; Puglisi, C.; Vitalini, D. J. Polym. Sci, Part A 1987, 25, 475-487.

5. Brown, R. S.; Wilkins, C. L. In Applications of Lasers in Polymer Science and Technology, Vol. 4; CRC: Boca Raton, FL (in press).

6. Bletsos, I. V.; Hercules, D. M.; Greifendorf, D.; Benninghoven, A. Anal. Chem. 1985, 57, 2384-2388.

7. Cochran, K. L. Appl. Spectrosc. Rev. 1986, 22, 137-187.

8. Doherty, S. J.; Busch, K. L. Anal. Chim. Acta 1986, 187, 117-127.

9. Mattern, D. E.; Hercules, D. M. Anal. Chem, 1985, 57, 2041-2046.

10. Lattimer, R. P.; Schulten, H. R. Int. J. Mass Spectrom. Ion Pracesses 1985, 67, 277-284.

11. Bletsos, I. V.; Hercules, D. M.; van Leyen, D.; Benninghoven, A. Macromolecules 1987, 20,407-413.

12. Gardella, I. A.; Hercules, D. M. Anal. Chem. 1980, 52, 226-232.

13. Brown, R. S.; Weil, D. A.; Wilkins, C. L. Macromolecules 1986 , 19, 1255-1260.

14. Nuwaysir, L. M.; Wilkins, C. L. Anal. Chem. 1988, 60, 279-282.

15. Ijames, C. F,; Wilkins, C. L. I. Am. Chem. Soc. 1988, 110, $2687-2688$.

16. Simonsick, W. J., Jr. J. Appl. Polm. Sci., Appl. Polym. Symp. 1989, 43, 257-274.

17. Bombick, D.; Allison, J. Anal. Chem. 1987, 59, 458466.

18. Simonsick, W. J., Jr. Proceedings of the 36 th ASMS Conference on Mass Spectrometry and Allied Topics; San Francisco, CA, June 1988, pp 470-471.

19. Cook, K. D.; Callahan, J. H.; Man, V. F. Anal. Chem. 1988, $60,706-713$.

20. Ghaderi, S.; Littlejohn, D. P. Proceedings of the 33rd ASMS Conference on Mass Spectrometry and Allied Topics; San Diego, CA, June 1985, pp 727-728.

21. Rempel, D. L.; Huang, S. K.; Gross, M. L. Int. J. Mass Spectrom. Ion Processes 1986, 70, 163-184.

22. Giancaspro, C.; Verdun, F, R. Anal. Chem. 1986, 58, 2097-2099.

23. Wise, M. B. Anal. Chem. 1987, 59, 2289-2293. 